\title{
Características psicológicas asociadas a la incidencia de lesiones en deportistas de modalidades individuales
}

\author{
Rosendo Berengüí-Gil ${ }^{1}$, Enrique J. Garcés de Los Fayos ${ }^{2}$ y María D. Hidalgo-Montesinos ${ }^{2}$ \\ ${ }^{1}$ Grados de Educación y Psicología. Universidad Católica San Antonio de Murcia \\ ${ }^{2}$ Facultad de Psicología. Universidad de Murcia
}

\begin{abstract}
Resumen: Las lesiones deportivas son una de las consecuencias más negativas que se derivan de la práctica deportiva. Son múltiples los factores que pueden estar en su origen, creciendo el interés en la búsqueda de las variables psicológicas que se encuentran asociados a su producción. En el presente estudio se evaluó a 84 deportistas de cuatro disciplinas individuales, de diferentes categorías de competición y nivel competitivo. Se registran 247 lesiones en el período de dos años, una media de 2.94 por deportista (1.47 por año), existiendo diferencias estadísticamente significativas entre las medias de lesiones de los cuatro deportes estudiados. Los deportistas de mayor edad sufren más lesiones, al igual que aquellos de mayor nivel de rendimiento. Mediante la administración del 16PF-5, IPED y SCAT, se obtiene que los deportistas con bajas puntuaciones en Estabilidad Emocional y Control de Afrontamiento Negativo, y altas en Ansiedad, presentan un mayor promedio de lesiones. Los sujetos sin lesiones se diferencian de los lesionados por mayores niveles de Estabilidad Emocional, Privacidad y Apertura al cambio, Control de Afrontamiento, y menores de Ansiedad. Existen correlaciones estadísticamente significativas entre el número de lesiones y las puntuaciones de Estabilidad Emocional, Tensión, Aprensión, Ansiedad, y ansiedad rasgo competitiva.

Palabras clave: Personalidad; variables psicológicas; lesiones deportivas; deporte competitivo; competición.
\end{abstract}

\section{Introducción}

Las lesiones son probablemente las consecuencias más negativas que se derivan de la práctica deportiva. La creciente inversión en horas de entrenamiento y las enormes cargas físicas y psicológicas que exige la competición al deportista suelen situar su organismo en condiciones extremas, y provocar un aumento en la vulnerabilidad a la lesión. A lo largo de décadas el foco de interés de la investigación ha estado centrado en exclusiva en los aspectos físicos de la lesión, ignorando los aspectos psicológicos. El minucioso examen de los fundamentos físicos y factores biomecánicos ha permitido el planteamiento de que esas variables por si solas no pueden dar explicación de todas las lesiones que acontecen (Andersen y Williams, 1999; Brewer, 2009; Smith, Ptacek y Smoll, 1992). Por ello se ha producido un aumento en la consideración, como básicos y relevantes, de los aspectos psicológicos y sociales, como el estrés, la presión ejercida por el entorno, o la personalidad del deportista, entre otros, en la producción de lesiones. De ahí nuestro interés por acercar al estudio de las lesiones deportivas las posibles relaciones que éstas guardan con los correlatos psicológicos.

Las lesiones pueden ser definidas y clasificadas de múltiples formas: en términos de los problemas médicos asociados, mediante la valoración de su gravedad, del tiempo de

* Dirección para correspondencia [Correspondence address]: Rosendo Berengüí Gil. Grados de Educación y Psicología. Universidad Católica San Antonio de Murcia. Campus de los Jerónimos, s/n. 30107 Murcia (España). E-mail: rberengui@ucam.edu
Title: Psychological characteristics associated with the incidence of sports injuries in athletes of individual sport.

Abstract: Sports injuries are one of the most negative consequences resulting from the practice of sports. There are many factors that may cause them, and right now there is growing interest in the psychological variables associated to their causes. The present study evaluated 84 athletes from four individual disciplines, different categories of competition and competitive level. 247 injuries were recorded during a two-year period with an average of 2.94 per athlete (1.47 per year). Statistically significant differences between the four studied sports injuries have been found. The older athletes suffered more injuries, as well as those with higher levels of performance. By administering the 16PF-5, IPED and SCAT, we find that athletes with low scores on Emotional Stability and Negative Energy Control, and high scores in Anxiety, have a higher injury rate. No injured subjects present a difference with the injured ones in their higher levels of Emotional Stability, Privateness and Openness to change, Control Coping, as well as in their lower Anxiety levels. Statistically significant correlations exist between the number of injuries and the athletes' scores in Emotional Stability, Tension, Apprehension, Anxiety, and competitive trait anxiety.

Key words: Personality; psychological variables; sports injuries; competitive sport; competition.

baja deportiva que conllevan o interrupción en la vida normal del lesionado, en términos de su frecuencia, y considerando la modificación en las actividades cotidianas del deportista que la lesión requiere (Noh, 2005). Una extendida y aceptada definición de lesión deportiva asume ésta como cualquier daño físico que lleva como resultado dolor o incomodidad, y que causa una o más de las siguientes condiciones: 1) la cesación de la actividad en una o más sesiones; 2) necesidad de modificar las actividades durante una o más sesiones; 3) efectos negativos en entrenamientos o actuaciones durante una o más ocasiones; y 4) suficiente angustia emocional que interfiera con la concentración o focalización durante una o más sesiones o actuaciones (Krasnow, Mainwaring y Kerr, 1999).

Se considera que las lesiones son producidas por una interacción compleja de eventos y múltiples factores de riesgo. Atendiendo a éstos, suelen considerarse dos categorías o bloques principales: factores externos o extrínsecos al deportista (como la conducta de los otros, factores deportivos propios de cada especialidad, o relacionados con el ambiente) y factores internos o intrínsecos del deportista, concretamente, médico-fisiológicos, biomecánicos y psicológicos. En la práctica deportiva se produce una interacción de ambos tipos de factores para provocar la susceptibilidad del deportista, antes de producirse el acontecimiento o evento incitador que provoca la ocurrencia de la lesión (Meeuwisse, Tyreman, Hagel y Emery, 2007).

Señalar además como cada deporte presenta una serie de rasgos y reglas propias y diferenciadoras, que conducen lógicamente a un patrón de lesiones particular de cada una de las 
disciplinas. Deportes como atletismo, ciclismo, piragüismo y taekwondo, analizados en el presente estudio, presentan unas características de competición que demandan del practicante unas cualidades físicas, técnicas, tácticas y psicológicas muy dispares, siendo responsables de la heterogeneidad en la casuística de sus lesiones.

Por otra parte, han sido planteados modelos explicativos que afirman la existencia de vínculos entre los aspectos psicológicos y la lesión, girando la mayoría alrededor del estrés como elemento fundamental que influye de muy diferentes formas sobre el individuo. Es el Modelo de Estrés y Lesión de Andersen y Williams (1988), y su posterior revisión (Williams y Andersen, 1998), la teoría multifactorial considerada como clave para comprender la relación entre las variables psicológicas y la lesión (Kolt, 2004). La respuesta de estrés es el núcleo central del modelo, al generar una serie de efectos perjudiciales sobre los mecanismos físicos o psicológicos del deportista como, por ejemplo, incrementos en la tensión muscular o déficits atencionales. Dicha respuesta es determinada y moderada por las relaciones bidireccionales entre variables como la personalidad del deportista, la historia de factores estresantes y sus recursos de afrontamiento. En el presente estudio se examina el papel de la personalidad global del deportista y de habilidades psicológicas fundamentales para el rendimiento deportivo, en la ocurrencia de las lesiones deportivas, considerando que la atención prestada a la misma ha sido limitada, especialmente en lo relativo al uso de cuestionarios generales de personalidad y su aplicación a deportistas de modalidades individuales.

A pesar de ese reducido empleo de cuestionarios de personalidad en este ámbito, encontramos estudios que han explorado la personalidad del deportista y factores aislados, además de múltiples variables constitutivas de la personalidad asumidas como relevantes para la comprensión del posible origen de las lesiones deportivas. Desde los estudios pioneros de Brown (1971) con futbolistas lesionados, han sido variados los intentos por delimitar las características del deportista fundamentales en el origen de la lesión. Así por ejemplo, empleando el 16PF, encontramos estudios que destacan como aquellos deportistas con un estilo de personalidad más dependiente, sensible y sobreprotegidos (Jackson et al., 1978), con menores niveles de privacidad (Taimela et al., 1990; Valliant, 1981), mayores de atrevimiento (Taimela et al., 1990), y valores elevados de ansiedad (Berengüí y Garcés de los Fayos, 2010; García y Aragües, 1998), padecen mayor número de lesiones. Yatabe et al. (2005) afirman que las diferencias en los rasgos de personalidad se consideran un riesgo de lesión ya que cada factor de personalidad se relaciona directamente con una respuesta emocional, e influyen en la incidencia de lesiones deportivas.

Además, el modelo original de Andersen y Williams (1988) proponía posibles variables de personalidad que moderarían la relación entre estrés y lesión. Entre ellas destaca el papel de la ansiedad rasgo competitiva. Un hecho a considerar es la contradicción en los resultados al revisar los estudios que han empleado medidas de ansiedad específicas del contexto deportivo y medidas de ansiedad general (Cassidy, 2006; Williams y Andersen, 2007). Así, mientras las investigaciones que han usado medidas generales no han encontrado relación entre ansiedad e incidencia de lesión, aquellas que han contado con instrumentos como el Sport Competition Anxiety Test, (SCAT; Martens, 1977), o Sport Anxiety Scale (SAS; Smith, Smoll y Schutz, 1990), sí proporcionan datos que confirman dicha asociación. En diferentes estudios (Berengüí, 2011; Blackwell y McCullagh, 1990; Hanson et al., 1992; Johnson e Ivarsson, 2010; Olmedilla, Andreu, Ortín y Blas, 2009; Passer y Seese, 1983; Petrie, 1993; Smith, Ptacek y Patterson, 2000) altas puntuaciones en ansiedad rasgo competitiva se ligaron a un mayor número de lesiones o mayor gravedad de las mismas. Es por ello que en el presente estudio, además de la evaluación de la ansiedad del deportista mediante la correspondiente dimensión global del $16 \mathrm{PF}-5$, se tiene en cuenta otra medida de ansiedad rasgo competitiva, en este caso el SCAT.

Señalar igualmente como la evaluación de variables o características psicológicas asociadas al rendimiento deportivo, y la relación de las mismas con las lesiones, han suscitado en los últimos años un creciente interés por parte de la investigación. Un mayor o menor nivel en dichas características puede conducir al óptimo o deficiente desempeño del deportista en el entrenamiento y las competiciones, y en consecuencia situarlo en riesgo de sufrir lesiones (Berengüí, López-Gullón, Garcés de los Fayos y Almarcha, 2011). En diferentes modalidades deportivas, variables como autoconfianza (Abenza, Olmedilla, Ortega y Esparza, 2009; Berengüí et al., 2011; Gimeno, Buceta y Pérez-Llantada, 2007; Olmedilla, 2005), concentración (Olmedilla, 2005) o habilidades de afrontamiento (Berengüí et al., 2011; Hanson, McCullagh y Tonymon, 1992; Maddison y Prapavessis, 2005; Ortín, 2008) han mostrado en mayor o menor medida su influencia sobre las lesiones.

Las elevadas cifras de lesiones que se registran anualmente, tanto en el deporte profesional como en el aficionado, requieren de un mayor volumen de investigación que estudie el papel desempeñado por los factores psicológicos en el origen de las lesiones deportivas. En los últimos años, a pesar de la prolífica producción científica (para una revisión véase Olmedilla, Ortega, Abenza y Boladeras, 2011), la inmensa mayoría de investigaciones centran sus objetivos en analizar las consecuencias psicológicas de la lesión deportiva y el comportamiento del lesionado en el camino a su plena recuperación, siendo escasos los estudios que analicen la relación entre factores psicológicos y la producción de lesiones. Además, entre éstos últimos, la gran mayoría de los mismos toman como muestra de estudio a deportistas de disciplinas de equipo (Abenza et al., 2009; Devantier, 2011; Ivarsson y Johnson, 2010; Johnson, 2011; Johnson, Ekengren y Andersen, 2005; Johnson e Ivarsson, 2011; Maddison y Prapavessis, 2005; Olmedilla, 2005; Ortín, 2008; Rogers y Landers, 2005; Steffen, Pensgaard y Bahr, 2009), siendo limitadas las referencias de investigaciones orientadas al estudio de deportes individuales (Berengüí, 2011; Berengüí et al., 
2011; Olmedilla, Prieto y Blas, 2011; Short, Reuter, Brandt, Short y Kontos, 2004; Stephan, Deroche, Brewer, Caudroit y La Scanff, 2009).

Los objetivos de este estudio son los siguientes: 1) conocer la incidencia de lesiones en deportistas de tecnificación deportiva de cuatro modalidades olímpicas individuales, y sus posibles diferencias en cuanto a sexo, disciplina practicada, categoría de competición y nivel competitivo, y 2) analizar la asociación entre personalidad, ansiedad rasgo competitiva y características psicológicas ligadas al rendimiento deportivo, con las lesiones deportivas.

\section{Método}

\section{Participantes}

La muestra estuvo compuesta por 84 sujetos, 50 hombres $(59.5 \%)$ y 34 mujeres $(40.5 \%)$, todos ellos pertenecientes a los programas de tecnificación deportiva (edades previas al paso a un deporte profesional o de alto rendimiento) de las federaciones de atletismo, ciclismo y piragüismo de la Región de Murcia (España), y de taekwondo y piragüismo de las federaciones españolas de esas modalidades. Todos ellos son deportistas de elevado nivel competitivo, ya que la mayor parte son finalistas en los campeonatos nacionales de sus disciplinas, y el $45.2 \%$ han integrado al menos en una ocasión sus respectivas selecciones nacionales.

La media de edad se sitúa en 18.30 años $(D T=1.74)$, con un rango de edades que abarca entre los 15 y 21 años. Debido a las diferencias de edades por categorías de competición en los distintos deportes decidimos dar uniformidad conformando tres grupos de edad. Así, por categorías de competición, 30 deportistas $(35.7 \%)$ son de categoría Cadete (hasta 16 años), 31 (36.9\%) de Junior (17 a 19 años), y 23 $(27.4 \%)$ de categoría Sub'21, deportistas de 20 y 21 años.

Por deportes, 22 sujetos son competidores de atletismo (26.2\%), 21 de ciclismo (25.0\%), 20 de piragüismo (23.8\%) y 21 de taekwondo (25.0\%). También se realiza una división según el nivel competitivo, quedando conformada la muestra según deportistas de nivel internacional (integrantes de sus respectivas selecciones nacionales) y de nivel nacional (finalistas en los campeonatos nacionales de sus disciplinas y participantes en torneos de alto nivel). Los deportistas internacionales son $38(45.2 \%)$ y 46 los de nivel nacional $(54.8 \%)$.

La media de edad de inicio en el deporte es de 10.23 (DT $=2.93)$, siendo de $6.68(D T=2.48)$ el promedio de años compitiendo en su disciplina. El mayor porcentaje de días de entrenamiento corresponde a quienes entrenan seis días a la semana $(54.8 \%)$, con una media de 5.46 días $(D T=.85)$. En cuanto a horas semanales de entrenamiento, el promedio es de 13.10 horas $(D T=4.03)$ por deportista.

\section{Instrumentos}

Sixteen Personality Factor Questionnaire, $5^{a}$ Edición (16PF-5). Cuestionario de personalidad con amplia tradición en su empleo en el ámbito deportivo. Para este estudio hemos utilizado su quinta edición (Cattell, Cattell y Cattell, 1995) que consta de 185 ítems, con un formato de respuesta de tres opciones. El instrumento surge de la investigación científica en los elementos básicos de la personalidad, y su estructura de rasgos proporciona una descripción rica e integrada de la persona, incluyendo los cinco grandes rasgos, siendo una medida comprensiva de la personalidad normal (Cattell y Schuerger, 2003).

El cuestionario está integrado por 16 escalas primarias, o rasgos de primer orden: Afabilidad (A), Razonamiento (B), Estabilidad (C), Dominancia (E), Animación (F), Atención a las normas $(\mathrm{G})$, Atrevimiento $(\mathrm{H})$, Sensibilidad (I), Vigilancia (L), Abstracción (M), Privacidad (N), Aprensión (O), Apertura al cambio (Q1), Autosuficiencia (Q2), Perfeccionismo (Q3) y Tensión (Q4). Las distintas combinaciones de esas escalas o rasgos de primer orden dan como resultado la obtención de cinco dimensiones globales de personalidad o factores de segundo orden: Extraversión, Ansiedad, Dureza, Independencia, y Autocontrol.

Respecto a ediciones anteriores incorpora tres escalas, o índices de estilo de respuesta (Aquiescencia, Manipulación de Imagen e Infrecuencia), y afirman Russell y Karol (2005) que en esta nueva versión se han mejorado las características psicométricas del instrumento, obteniendo una fiabilidad promedio de .74 .

Inventario Psicológico de Ejecución Deportiva (IPED). Adaptación de la escala Psychological Performance Inventory (PPI) de Loehr (1986). Compuesto por 42 ítems con escala de respuesta tipo Likert de cinco opciones (casi siempre, a menudo, a veces, rara vez, y casi nunca). En el presente trabajo empleamos la versión en castellano a partir del trabajo de adaptación y baremación de Hernández (2006) con poblaciones deportivas españolas. Integra siete subescalas:

1. Autoconfianza (AC): ítems relativos a cogniciones y sentimientos positivos que indican el grado de certeza que tiene el deportista respecto a su habilidad para alcanzar el éxito en una determinada tarea.

2. Control de Afrontamiento Negativo (CAN): escala referente al control por parte del deportista de emociones negativas, como el miedo, ansiedad, rabia, frustración, etc.

3. Control de la Atención (CAT): señala la capacidad de percibir y asimilar de forma apropiada la información que transmiten entrenador y entorno, y detección de estímulos significativos de la situación deportiva.

4. Control Visual e Imaginativo (CVI): capacidad de controlar situaciones y experiencias a nivel mental.

5. Nivel Motivacional (NM): partiendo de su importancia como motor del comportamiento, sus ítems incluyen cuestiones como el grado de compromiso e interés del deportista por su deporte, importancia que le concede, o su disposición y afrontamiento respecto al entrenamiento y superación diarios.

6. Control de Afrontamiento Positivo (CAP): es un indicador de la capacidad del deportista para buscar la dinami- 
zación a través de emociones positivas, al tiempo que se mantengan controladas las negativas.

7. Control Actitudinal (CAT): dominio sobre la predisposición para la acción y para la clasificación de los objetos, personas y conductas del entorno del individuo, y sobre el grado de reacción ante éstos y su consistencia evaluativa.

Las escalas son unidimensionales y presentan valores de fiabilidad entre .61 y .75 (Hernández, 2006).

Sport Competition Anxiety Test (SCAT). El test de Martens (1977) y Martens, Vealley y Burton (1990) es una medida específica de ansiedad rasgo en el contexto deportivo que evalúa la tendencia individual a percibir las situaciones competitivas como amenazantes y para responder a esas situaciones con sentimientos de aprensión y tensión. Conformado por 15 ítems, con tres opciones de respuesta, obteniéndose una puntuación directa de ansiedad rasgo que oscila entre 10 y 30. Existen versiones paralelas del instrumento para niños (10 a 14 años) y para adultos (15 años en adelante), modelo A, siendo ésta última la empleada en nuestro estudio. Los autores confirman unos valores de consistencia interna entre .95 y .97 (Martens et al., 1990) y, en el contexto del estudio de lesiones, diferentes trabajos han hecho uso del cuestionario obteniendo coeficientes de fiabilidad (alpha de Cronbach) de .84 (Ortín, 2008) y .85 (Berengüí, 2011).

Cuestionario sociodemográfico y de lesiones. Para el registro de los datos personales y deportivos se utiliza un cuestionario ad hoc (ver Anexo), mediante el cual también se obtiene la información relevante de lesiones para el estudio. Se definieron las lesiones que debían ser registradas como aquellas acontecidas como resultado de la participación en un entrenamiento o competición, y que implicaron la restricción o el cese completo de la participación del deportista un día o más a partir del día de inicio de la lesión (Hootman, Dick y Agel, 2007). En el cuestionario se solicitaba que el deportista detallara las lesiones sufridas durante las dos últimas temporadas y el tiempo (en días) transcurrido desde el inicio hasta la plena recuperación de cada una de las lesiones. También se requería una breve explicación sobre cuál fue la causa de la lesión, para así poder decidir sobre la inclusión de aquellas directamente relacionadas con la práctica deportiva o descartar lesiones que fueran ajenas a la misma.

\section{Procedimiento}

Previo al comienzo del estudio se contactó con los directores técnicos y responsables de las distintas federaciones, que fueron informados de los objetivos del estudio y qué características debían reunir los deportistas participantes (integrantes de programas de tecnificación deportiva, de ambos sexos y entre 15 y 21 años). Tras su conformidad, se concertaron fechas para poder tomar contacto con los deportistas y entrenadores, y acudir a concentraciones técnicas de cada disciplina en las cuáles las pruebas fueron administradas. En las citadas concentraciones, antes de suministrar los cuestio- narios se informaba a los deportistas del propósito y objetivos del estudio, el tratamiento de confidencialidad que recibirían los datos aportados, y se firmó su consentimiento.

Tras cumplimentar los cuestionarios se contrastó la información que aportaron los deportistas respecto a las lesiones con el criterio de los entrenadores, para poder atender posibles errores u omisiones en la cumplimentación del registro. Finalmente, las lesiones registradas fueron analizadas en reuniones periódicas por colaboradores especialistas en medicina y fisioterapia deportivas, para así poder analizar dudas o incorrecciones, haciendo uso del sistema OSICS (Orchard Sports Injury Classification System; Rae y Orchard, 2007) para codificar las lesiones y realizar un adecuado tratamiento y clasificación de las mismas.

\section{Diseño y análisis estadístico}

Se llevó a cabo un estudio descriptivo transversal, utilizando metodología cuantitativa.

Se emplearon métodos estadísticos estándar para el cálculo de las medias y la desviación típica (DT). Se conformaron grupos para analizar las diferencias de medias en lesiones entre sujetos con puntuaciones bajas y altas en los cuestionarios de evaluación. Para ello se utilizó el criterio de restar (grupo bajo) o sumar (grupo alto) una desviación típica a la media. Para examinar esas diferencias entre grupos se empleó la prueba $t$ de Student para muestras independientes. También se utiliza análisis de varianza (ANOVA) para analizar las diferencias entre grupos de lesiones en las diferentes escalas de evaluación. Para ello se ha realizado una división de los deportistas en cinco grupos dependiendo del número concreto de lesiones registradas (ninguna lesión, una, dos, tres, y cuatro ó más lesiones). Se emplea también ANOVA para comparar los datos de las distintas variables deportivas. Asimismo se efectúa un análisis de correlación (coeficiente de Pearson) para observar las pautas de variación común entre las puntuaciones en las escalas psicológicas y el número de lesiones registradas.

En todo caso se consideró un nivel de significación estadística del 5\% $(p \leq .05)$. Para este análisis estadístico se empleó el paquete informático SPSS para Windows (versión 17.0, SPSS Inc.).

\section{Resultados}

Se registran un total de 247 lesiones en el periodo de estudio, que supone una media de $2.94(D T=2.481)$ por deportista (1.47 por año). El rango de lesiones varía entre ninguna lesión (12 casos, 14.3\%) y diez lesiones ( 2 sujetos, $2.4 \%$ ). El mayor porcentaje corresponde a quienes han sufrido una $\mathrm{O}$ tres lesiones, en ambos $21.4 \%$ (18 sujetos).

La media de lesiones en hombres es $3.28(D T=2.741)$ y $2.44(D T=1.972)$ en mujeres, no existiendo diferencias entre sexos. Por deportes (Tabla 1), atletismo es la modalidad con menor número de lesiones (33), siendo su media de 1.50 $(D T=1.596)$. Mientras en el extremo opuesto taekwondo 
presenta las mayores cifras, con un total de 97 lesiones $(39.28 \%)$ y promedio por deportista de $4.62(D T=2.747)$. Existen diferencias estadísticamente significativas entre deportes $\left(F_{(3,80)}=7.428 ; p<.001\right)$. Al realizar comparaciones post hoc, resultan estadísticamente significativas $(p<.05)$ las diferencias de medias entre atletismo y piragüismo, atletismo y taekwondo, y entre ciclismo y taekwondo.

Tabla 1. Distribución de lesiones por deportes.

\begin{tabular}{llllll}
\hline Deporte & Lesiones & Porcentaje & Rango & Media & DT \\
\hline Atletismo & 33 & 13.36 & $0-6$ & 1.50 & 1.596 \\
Ciclismo & 52 & 21.05 & $0-5$ & 2.48 & 1.692 \\
Piragüismo & 65 & 26.31 & $0-9$ & 3.25 & 2.693 \\
Taekwondo & 97 & 39.28 & $0-10$ & 4.62 & 2.747 \\
Total & 247 & $100 \%$ & $0-10$ & 2.94 & 2.481 \\
\hline
\end{tabular}

Del mismo modo se encuentran diferencias estadísticamente significativas en el análisis por categorías de competición $\left(F_{(2,81)}=7.267 ; p<.001\right)$. Los deportistas de categoría Cadete promedian 2.03 lesiones $(D T=1.935)$ y $2.71(D T=$ 2.571) los de Junior, encontrándose dichas diferencias entre estos dos grupos respecto a Sub'21, con una media de 4.43 lesiones $(D T=3.021)$. En el análisis por nivel competitivo, los deportistas de nivel Nacional presentan una media de 2.22 lesiones $(D T=1.823)$ frente a $3.82(D T=2.922)$ de los competidores de nivel Internacional, existiendo diferencias significativas $\left(t_{82}=-3.087 ; p=.003\right)$.

Tabla 2. Medias y desviaciones típicas de la muestra en los cuestionarios psicológicos.

\begin{tabular}{|c|c|c|c|c|c|c|c|}
\hline \multicolumn{4}{|c|}{ Escalas 16PF-5 } & \multicolumn{4}{|c|}{ Dimensiones 16PF-5 (Decatipos) } \\
\hline & Min & Máx & $M(D T)$ & & Min & Máx & $M(D T)$ \\
\hline A & 5 & 22 & $13.62(4.08)$ & EXT & 0 & 10 & $4.52(1.93)$ \\
\hline B & 2 & 15 & $8.86(2.69)$ & ANS & 2 & 10 & $5.88(1.91)$ \\
\hline$C$ & 2 & 20 & $13.11(4.38)$ & DUR & 2 & 10 & $6.33(1.81)$ \\
\hline $\mathrm{E}$ & 5 & 20 & $13.30(3.68)$ & IND & 2 & 10 & $5.35(1.98)$ \\
\hline $\mathrm{F}$ & 2 & 20 & $13.67(3.86)$ & $\mathrm{CON}$ & 1 & 10 & $4.68(2.07)$ \\
\hline G & 4 & 22 & $13.11(4.30)$ & IPED & & & \\
\hline $\mathrm{H}$ & 2 & 20 & $12.98(5.43)$ & $\overline{\mathrm{AC}}$ & 9 & 30 & $23.75(4.26)$ \\
\hline & 0 & 20 & $8.83(4.50)$ & CAN & 11 & 28 & $20.46(3.94)$ \\
\hline L & 0 & 19 & $10.77(4.21)$ & CAT & 14 & 30 & $22.30(3.54)$ \\
\hline M & 0 & 18 & $6.82(4.37)$ & CVI & 9 & 30 & $24.11(4.44)$ \\
\hline $\mathrm{N}$ & 0 & 20 & $11.12(4.73)$ & NM & 14 & 30 & $25.48(3.23)$ \\
\hline $\mathrm{O}$ & 0 & 20 & $11.62(4.59)$ & CAP & 13 & 30 & $24.19(3.30)$ \\
\hline Q1 & 1 & 28 & $14.32(4.64)$ & $\mathrm{ACT}$ & 14 & 30 & $23.51(3.69)$ \\
\hline Q2 & 0 & 16 & $5.55(4.05)$ & SCAT & & & \\
\hline Q3 & 4 & 20 & $13.39(3.99)$ & SCAT & 13 & 30 & $22.21(4.51)$ \\
\hline Q4 & 0 & 20 & $10.75(5.08)$ & & & & \\
\hline
\end{tabular}

En la Tabla 2 se recogen los descriptores básicos de las distintas escalas de evaluación en la muestra. A partir de esos datos se conforman dos grupos, para comparar las lesiones de los grupos con puntuaciones bajas y altas en las escalas de evaluación psicológicas. En el análisis del 16PF-5 (Tabla 3) se observan diferencias estadísticamente significativas en la escala C o Estabilidad Emocional ( $t_{28}=2.848 ; p=.008$ ), ya que quienes conforman el grupo de bajas puntuaciones en la misma promedian 4.69 lesiones frente a 2.07 del grupo de al- tas puntuaciones. También se encuentran esas diferencias en la dimensión global Ansiedad, con una media de 3.94 lesiones en el grupo alto, por 2.03 del bajo. Se observa tendencia a la significación estadística en la escala Aprensión $\left(t_{24}=\right.$ $1.880 ; p=.070)$.

Tabla 3. Grupos con puntuaciones bajas o altas en las escalas y dimensiones del $16 \mathrm{PF}-5$ medias de lesiones y prueba $t$

\begin{tabular}{llllll} 
& \multicolumn{3}{c}{ Bajo } & \multicolumn{3}{c}{ Alto } & \\
\cline { 2 - 5 } & $n$ & $M(D T)$ & $n$ & $M(D T)$ & $t$ \\
A & 15 & $2.13(2.36)$ & 18 & $3.22(1.93)$ & $t_{31}=-1.461 ; p=.154$ \\
B & 17 & $2.00(1.37)$ & 15 & $2.20(2.30)$ & $t_{30}=-.303 ; p=.764$ \\
C & 16 & $4.69(3.09)$ & 14 & $2.07(1.59)$ & $t_{28}=2.848 ; p=.008$ \\
E & 12 & $3.33(3.05)$ & 17 & $2.88(2.80)$ & $t_{27}=.411 ; p=.684$ \\
F & 12 & $3.33(2.57)$ & 14 & $2.57(2.17)$ & $t_{24}=.819 ; p=.421$ \\
G & 20 & $2.55(2.60)$ & 14 & $2.71(1.38)$ & $t_{32}=-.215 ; p=.831$ \\
H & 13 & $3.62(2.56)$ & 11 & $2.45(1.81)$ & $t_{22}=1.257 ; p=.222$ \\
I & 17 & $3.06(2.92)$ & 15 & $2.80(1.69)$ & $t_{30}=.301 ; p=.766$ \\
L & 14 & $2.93(1.90)$ & 13 & $3.15(2.48)$ & $t_{25}=-.266 ; p=.792$ \\
M & 17 & $2.24(1.82)$ & 14 & $2.43(2.50)$ & $t_{29}=-.249 ; p=.805$ \\
N & 18 & $2.83(2.30)$ & 20 & $2.25(2.24)$ & $t_{36}=.789 ; p=.435$ \\
O & 13 & $2.00(1.47)$ & 8 & $3.75(2.81)$ & $t_{24}=-1.880 ; p=.070$ \\
Q1 & 7 & $2.57(1.51)$ & 12 & $2.25(2.73)$ & $t_{17}=.284 ; p=.779$ \\
Q2 & 12 & $2.17(1.80)$ & 15 & $3.27(2.86)$ & $t_{25}=-1.157 ; p=.258$ \\
Q3 & 14 & $3.43(3.69)$ & 17 & $2.47(1.97)$ & $t_{29}=.923 p=.363$ \\
Q4 & 10 & $2.70(1.49)$ & 18 & $3.50(2.99)$ & $t_{26}=-.787 ; p=.438$ \\
\hline EXT & 22 & $2,73(2.64)$ & 4 & $3.00(1.63)$ & $t_{24}=-.198 ; p=.845$ \\
ANS & 12 & $2.03(2.01)$ & 20 & $3.94(2.60)$ & $t_{30}=-1.994 ; p=.048$ \\
DUR & 8 & $2.63(2.77)$ & 21 & $3.78(2.76)$ & $t_{27}=-.740 ; p=.466$ \\
IND & 11 & $3.55(2.66)$ & 12 & $3.33(3.17)$ & $t_{21}=.173 ; p=.864$ \\
CON & 25 & $2.44(2.80)$ & 10 & $3.10(1.45)$ & $t_{33}=-.704 ; p=.486$ \\
\hline
\end{tabular}

Por su parte en el análisis del IPED (Tabla 4) las diferencias son encontradas en la escala Control de Afrontamiento Negativo $\left(t_{47}=1.946 ; p=.037\right)$, ya que los deportistas con bajo afrontamiento de emociones negativas presentan una media de 3.99 lesiones, por 2.15 del grupo con altas puntuaciones en la escala. También existen claramente esas diferencias en ansiedad rasgo competitiva $\left(t_{25}=-2.936 ; p=\right.$ .007), ya que quienes puntúan bajo en la prueba registran menos lesiones (1.90) que aquellos con altas puntuaciones (4.33).

Tabla 4. Grupos con puntuaciones bajas o altas en las escalas del IPED y SCAT: medias de lesiones y prueba $t$

\begin{tabular}{llllll}
\hline & \multicolumn{3}{c}{ Bajo } & \multicolumn{3}{c}{ Alto } & \multirow{2}{*}{$t$} \\
\cline { 2 - 5 } & $n$ & $M(D T)$ & $n$ & $M(D T)$ & \\
\hline AC & 28 & $3.13(2.33)$ & 16 & $2.75(2.53)$ & $t_{42}=.486 ; p=.630$ \\
CAN & 26 & $3.99(2.69)$ & 23 & $2.15(1.92)$ & $t_{47}=1.946 ; p=.037$ \\
CAT & 27 & $2.89(2.69)$ & 25 & $2.80(2.12)$ & $t_{50}=.131 ; p=.896$ \\
CVI & 13 & $3.69(2.01)$ & 37 & $2.89(2.63)$ & $t_{48}=.996 ; p=.630$ \\
NM & 14 & $2.29(1.93)$ & 16 & $3.69(3.26)$ & $t_{28}=-1.404 ; p=.121$ \\
CAP & 9 & $3.44(1.74)$ & 17 & $3.18(3.16)$ & $t_{24}=.234 ; p=.817$ \\
ACT & 12 & $2.75(1.96)$ & 11 & $2.91(3.20)$ & $t_{21}=-.145 ; p=.886$ \\
\hline SCAT & 16 & $1.81(1.90)$ & 11 & $4.33(3.26)$ & $t_{25}=-2.936 ; p=.007$ \\
\hline
\end{tabular}

A continuación se realiza el contraste de medias mediante la comparación de grupos de lesiones. En la Tabla 5 puedecomprobarse la distribución de los deportistas en los mismos. El mayor porcentaje corresponde al grupo de 4 ó 
más lesiones, el 30.95\%, mientras que el grupo de 2 lesiones constituye el $11.90 \%$, el menor.

Tabla 5. Distribución de deportistas en grupos de lesiones.

\begin{tabular}{lll}
\hline Grupo de lesiones & Deportistas & $\%$ \\
\hline 0 & 12 & 14.29 \\
1 & 18 & 21.43 \\
2 & 10 & 11.90 \\
3 & 18 & 21.43 \\
4 ó más & 26 & 30.95 \\
\hline
\end{tabular}

Las medias de lesiones y desviaciones típicas en cada uno de los grupos, en las distintas escalas y dimensiones globales del $16 \mathrm{PF}-5$ se recogen la Tabla 6. Encontramos diferencias estadísticamente significativas en varias escalas. En primer lugar, en Estabilidad Emocional o $C\left(F_{(4,79)}=3.360 ; p<.01\right)$, donde se comprueba un descenso de puntuaciones conforme aumenta el número de lesiones. Mediante comparaciones post hoc, resultan estadísticamente significativas $(p<.05)$ las diferencias de medias de los grupos sin lesiones, una y dos lesiones, y las medias de los grupos de tres y cuatro lesiones. También en N o Privacidad $\left(F_{(4,79)}=2.137 ; p=<.05\right)$, con mayores puntuaciones en el grupo de no lesionados que el grupo de cuatro o más lesiones $(p<.05)$. En la escala Q1 o Apertura al cambio resultan estadísticamente significativas $\left(F_{(4,79)}=2.858 ; p<.05\right)$ las diferencias de medias del grupo sin lesiones y las del resto de grupos. En cuanto a la dimensión Ansiedad $\left(F_{(4,79)}=2.653 ; p<.05\right)$ las diferencias se localizan entre el grupo sin lesiones, con media de 5.00, y los grupos de tres (6.67) y cuatro lesiones (6.31).

Tabla 6. Medias y desviaciones típicas de los grupos de lesiones en el 16PF-5.

\begin{tabular}{|c|c|c|c|c|c|}
\hline & Sin lesión & Una lesión & Dos lesiones & Tres lesiones & Cuatro ó más \\
\hline$\overline{\mathrm{A}}$ & $13.00(4.26)$ & $12.28(3.92)$ & $14.20(5.20)$ & $13.67(4.20)$ & $14.58(3.53)$ \\
\hline B & $9.25(2.86)$ & $9.28(2.69)$ & $9.50(3.44)$ & $7.78(3.02)$ & 8.88 (1.95) \\
\hline $\mathrm{C}$ & $15.40(3.24)$ & $15.17(3.95)$ & $13.94(3.60)$ & $12.19(4.82)$ & $11.06(4.25)$ \\
\hline $\mathrm{E}$ & $14.50(4.48)$ & $12.83(3.09)$ & $14.20(4,24)$ & $12.33(2.83)$ & $13.38(3.99)$ \\
\hline F & $14.25(2.86)$ & $14.17(3,54)$ & $12.20(5.22)$ & $13.56(3.72)$ & $13.69(4.12)$ \\
\hline G & $11.75(3.91)$ & $12.61(5.11)$ & $13.70(5.42)$ & $13.39(3.74)$ & $13.65(3.90)$ \\
\hline $\mathrm{H}$ & $14.83(5.24)$ & $11.39(5.10)$ & $15.50(3.50)$ & $12.17(5.62)$ & $12.81(5.99)$ \\
\hline $\mathrm{I}$ & $9.75(5.45)$ & $7.00(3.53)$ & $9.60(4.57)$ & $9.50(3.63)$ & $8.92(5.09)$ \\
\hline $\mathrm{L}$ & $10.42(3.37)$ & $11.33(4.56)$ & $11.00(5.29)$ & $10.78(4.87)$ & $10.46(3.63)$ \\
\hline M & $6.67(3.77)$ & $6.11(4.64)$ & $6.70(6.01)$ & $8.17(4.16)$ & $6.50(3.99)$ \\
\hline $\mathrm{N}$ & $12.92(5.18)$ & 11.61 (4.98) & $10.20(5.28)$ & $10.67(4.10)$ & $9.62(4.12)$ \\
\hline $\mathrm{O}$ & $11.00(4.65)$ & $10.83(4.42)$ & $10.10(6.33)$ & $12.44(3.92)$ & $12.46(4.43)$ \\
\hline Q1 & $18.08(5.76)$ & $13.67(3.78)$ & $12.70(5.49)$ & $13.22(2.46)$ & $14.42(4.80)$ \\
\hline Q2 & $4.08(4.79)$ & $6.89(3.94)$ & $5.30(3.92)$ & $5.39(4.23)$ & $5.50(3.71)$ \\
\hline Q3 & $12.83(3.92)$ & $13.22(4.25)$ & $12.40(5.14)$ & $14.67(3.16)$ & $13.27(3.97)$ \\
\hline Q4 & $10.83(5.41)$ & $9.33(4.66)$ & $10.00(6.09)$ & $11.22(3.99)$ & $11.65(5.60)$ \\
\hline$\overline{\mathrm{EXT}}$ & $4.00(2.00)$ & $3.94(2.01)$ & $4.70(2.11)$ & $4.78(2.07)$ & $4.92(1.67)$ \\
\hline ANS & $5.00(1.48)$ & 5.61 (1.85) & $5.90(2.60)$ & $6.67(1.37)$ & $6.31(1.93)$ \\
\hline DUR & 5.42 (1.97) & $6.67(1.71)$ & $6.30(2.21)$ & $6.78(1.51)$ & $6.23(1.79)$ \\
\hline IND & $6.58(2.11)$ & 5.17 (1.54) & $5.60(1.89)$ & $4.83(1.42)$ & $5.15(2.41)$ \\
\hline $\mathrm{CON}$ & $4.25(1.66)$ & $4.06(2.33)$ & $4.80(2.53)$ & $5.22(2.13)$ & $4.88(1.81)$ \\
\hline
\end{tabular}

En el Inventario Psicológico de Ejecución Deportiva (Tabla 7), se encuentran diferencias en la escala Control de Afrontamiento Negativo $\left(F_{(4,79)}=2.439 ; p<.05\right)$, resultando estadísticamente significativas $(p<.05)$ las diferencias de medias del grupo sin lesiones (21.58) respecto a tres (18.28) y cuatro lesiones (18.29), y de una lesión (21.83) con los mismos grupos. También existen diferencias estadísticamente significativas en el SCAT $\left(F_{(4,79)}=4.164 ; p<.01\right)$, en concreto entre el grupo de no lesionados (19.17) y los de tres (22.94) y cuatro ó más lesiones (25.08), y entre una (21.72) y cuatro ó más lesiones.

Tabla 7. Media y desviaciones típicas de los grupos de lesiones en el IPED y SCAT.

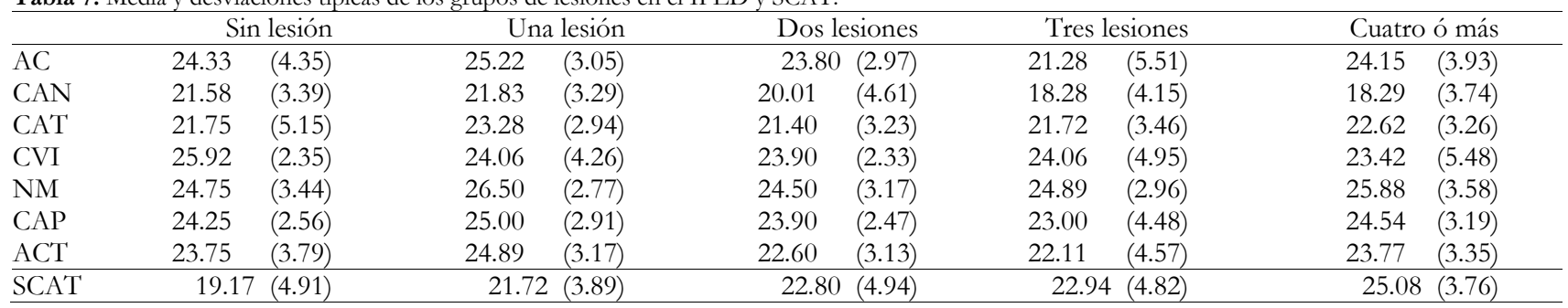


Por último se realizó un análisis de correlaciones entre el número de lesiones y las distintas escalas de los cuestionarios. Se registraron correlaciones estadísticamente significativas, aunque de pequeña magnitud, entre ese número de lesiones y varias escalas del 16PF-5, en concreto, con Estabilidad Emocional $(r=-.352 ; p=.001)$, Q4 o Tensión $(r=.332$; $p=.006)$, y Aprensión $(\mathrm{O})(r=.230 ; p=.036)$, además de la dimensión Ansiedad $(r=.370 ; p<.000)$. La correlación es de magnitud moderada en el SCAT $(r=.412 ; p<.000)$

\section{Discusión}

Mediante el presente estudio se ha buscado conocer, por un lado, la incidencia de lesiones en deportistas de tecnificación deportiva de cuatro modalidades olímpicas individuales (atletismo, ciclismo, piragüismo y taekwondo), y analizar las diferencias por sexo, modalidad, categoría de competición y nivel competitivo. En segundo lugar, se pretende analizar las relaciones de la personalidad del deportista, ansiedad rasgo competitiva y características psicológicas ligadas al rendimiento deportivo, con el padecimiento de lesiones deportivas.

Respecto al análisis de lesiones, muchos de los resultados encontrados siguen la línea de estudios previos. Así, existen diferencias entre deportes, sobre todo respecto a taekwondo. Las disciplinas de combate por sus propias características competitivas, y en especial el taekwondo, suelen situarse como aquellas con mayor número o más alto índice de lesiones, hecho constatado en numerosas investigaciones (Berengüí, 2011; Berengüí et al., 2011; Kazemi y Pieter, 2004; Pieter y Zemper, 1999; Zetaruk, Violán, Zurakowski y Micheli, 2005). También son constatadas diferencias entre categorías de competición, registrando los deportistas de mayor edad (Sub'21) mayor promedio de lesiones, en consonancia con los resultados de Berengüí (2011), Dvorak y Junge (2000), Junge y Dvorak (2004) y Stevenson, Hamer, Finch, Elliot y Kresnow (2000). La principal explicación puede estar al atender a factores de riesgo básicos para la lesión, como la creciente inversión en horas de entrenamiento o el incremento de exigencias a nivel físico y psicológico, los cuales acarrean al deportista una mayor exposición a los factores de riesgo y, por tanto, más oportunidades para lesionarse. Las diferencias de igual forma son halladas en cuanto al nivel competitivo del deportista, ya que los deportistas de nivel internacional presentan un índice superior. Al igual que con la anterior variable debe contemplarse que un nivel más alto de rendimiento lleva asociada una mayor inversión de recursos personales por parte del deportista que conducen a una mayor exposición a factores de riesgo y, por tanto, un mayor riesgo de lesión. Sin embargo, y a diferencia de otros estudios (Berengüí, 2011; Berengüí, Garcés de Los Fayos, Almarcha y Ortega, 2010) no se localizan diferencias entre hombres y mujeres.

En cuanto al objetivo principal del estudio, la relación de las diferentes variables psicológicas con la ocurrencia de lesiones, podemos comprobar a la luz de los resultados halla- dos en este estudio cómo los datos señalan relaciones entre los niveles de determinadas características psicológicas y el padecimiento de lesiones.

Los deportistas con menores niveles en Estabilidad Emocional registran mayor número de lesiones deportivas, encontrando asimismo tendencia a la significación estadística en Aprensión, donde los deportistas con mayores niveles registran más lesiones que los sujetos menos aprensivos. Ambas escalas contribuyen a las puntuaciones de la dimensión Ansiedad, también significativa. La escala Estabilidad Emocional evalúa la estabilidad del sujeto y la forma de afrontar sus problemas cotidianos. Puntuaciones altas definen a una persona emocionalmente estable, adaptable y madura, mientras puntuaciones bajas lo hacen de sujetos emocionalmente cambiables y reactivos (Karson, Karson y O'Dell, 2003; Russell y Karol, 2005). Por su parte, Aprensión es una escala relacionada con la autoestima, el experimentar culpa e inseguridades, mostrándose los sujetos del polo bajo seguros, despreocupados y satisfechos, mientras el polo alto describe a aprensivos, dubitativos y preocupados. Al mismo tiempo, Ansiedad es definitoria de personas ajustadas (en su polo bajo, estables emocionalmente, confiadas, seguras y relajadas) frente a personas ansiosas (reactivas, suspicaces, aprensivas y tensas). Asimismo, hemos podido comprobar que en Estabilidad Emocional se produce un descenso de puntuaciones conforme aumenta el número de lesiones, a través de los grupos conformados, y en Ansiedad todo lo contrario, esto es, un aumento de puntuaciones conforme se avanza en grupos de lesiones.

Estos resultados encuentran respaldo en estudios anteriores (Berengüí, 2011; Blackwell y McCullagh, 1990; Hanson et al., 1992; Johnson e Ivarsson, 2010; Olmedilla et al., 2009; Passer y Seese, 1983; Petrie, 1993; Smith, Ptacek y Patterson, 2000), ya que altas puntuaciones en ansiedad rasgo estuvieron unidas a un mayor número de lesiones o mayor gravedad de las mismas. En su estudio, García y Aragües (1998), empleando el 16-PF en jugadores de fútbol, hallan asociación entre valores altos de ansiedad y el padecimiento de lesiones, sosteniendo que cualquier puntuación extrema en ansiedad, tanto por exceso como por defecto, es un factor favorecedor para el padecimiento de las mismas. También los resultados parecen seguir los planteamientos de Williams y Andersen (1998) al considerar que altos niveles de ansiedad (y características asociadas como las apuntadas de estabilidad y aprensión, en el presente estudio) pueden desequilibrar las apropiadas condiciones fisiológicas y de concentración necesarias para una correcta ejecución, y llevar ligado inevitablemente un aumento en la vulnerabilidad a la lesión. Señalar como, en el caso de la ansiedad, los datos son estadísticamente significativos tanto con el empleo de una escala no procedente del ámbito deportivo, como de una derivada del mismo.

A lo anterior habría que sumar los datos encontrados en cuanto a correlaciones estadísticamente significativas, concretamente, se observa una tendencia al aumento de las lesiones conforme aumentan las puntuaciones en Aprensión, 
Tensión y Ansiedad, del 16PF-5, y ansiedad rasgo competitiva del SCAT, además de una correlación significativa de signo negativo con Estabilidad Emocional. En esa línea Berengüí y Garcés de los Fayos (2010) hallan correlación positiva entre el número de lesiones y la dimensión global Ansiedad del 16PF-5, y también, y mediante el EPQ de Eysenck, Berengüí y Garcés de los Fayos (2008) obtienen correlación de mayor magnitud entre ese número de lesiones y la dimensión Neuroticismo. Consideran Russell y Karol (2005) que, frente a personas ajustadas, las ansiosas se caracterizan por ser reactivas, suspicaces, aprensivas y tensas, experimentan con mayor frecuencia afectos negativos, presentan dificultad para controlar adecuadamente sus emociones o reacciones, y pueden actuar con conductas contraproducentes. En vista de esta descripción resulta evidente que los deportistas así caracterizados puedan albergar estados afectivos y de activación física y psicológica que sitúen su organismo en una posición de riesgo para padecer las lesiones, por la ausencia del pertinente ajuste global que demanda la ejecución deportiva.

Otros resultados interesantes, coincidentes con una investigación anterior (Berengüí, 2011), son las diferencias halladas entre el grupo de deportistas sin lesiones y el resto de grupos en sus puntuaciones en Apertura al cambio y Privacidad. En ambas los sujetos no lesionados presentan medias superiores. Altas puntuaciones en Apertura al cambio indican el interés por la innovación, creatividad, flexibilidad y adaptación a las nuevas circunstancias, y es por ello que esa orientación a una mayor apertura poríamos asumirla como protectora ante la lesión. Las características definitorias expuestas del polo alto de Apertura al cambio pueden contemplarse como positivas para afrontar las demandas deportivas, más que las descriptoras del polo opuesto, es decir, resistencia al cambio, rigidez o incapacidad para adaptarse a las nuevas circunstancias. Respecto a Privacidad, ésta describe a personas abiertas, genuinas, llanas y naturales, frente a privadas, calculadoras, discretas y que no se abren a los demás. En Valliant (1981) y Taimela et al. (1990) encontramos los únicos estudios que han localizado diferencias respecto a las lesiones, y en ambos se confirman, puntuaciones inferiores en Privacidad en deportistas lesionados.

Entre las variables psicológicas relacionadas con el rendimiento deportivo destaca principalmente Control de Afrontamiento Negativo. Obtenemos una media de lesiones mucho más alta entre quienes puntúan bajo en esta escala, y puntuaciones mayores en la escala de los deportistas sin lesión o una, que el resto de grupos. Loehr (1986), el autor del cuestionario utilizado (IPED), describe la escala como una habilidad para controlar emociones negativas como ansiedad, miedo o frustración, y su control es esencial para el éxito deportivo, por tanto conviene mantenerse calmado, relajado y concentrado para mantener la energía negativa al mínimo. El control de esa energía está unido a la habilidad de percibir las situaciones difíciles como un desafío en vez de como una amenaza o problema. El afrontamiento en psicología del deporte goza de una posición central. Para Lazarus y Folkman (1984) supone aquellos esfuerzos cognitivos y conductuales constantemente cambiantes que se desarrollan para manejar las demandas específicas externas y/o internas que son evaluadas como excedentes o desbordantes de los recursos del individuo. El contexto deportivo, en especial la competición, obliga al deportista a hacer frente a múltiples de esas demandas o estresores específicos, que originan multitud de reacciones, como elevada ansiedad, déficits de concentración o, en ocasiones, un decremento en la confianza del deportista sobre sus capacidades. Factores como los anteriores lógicamente pueden conducir a actuaciones defectuosas que produzcan las lesiones.

Los principales modelos psicológicos explicativos del proceso de lesión asumen, entre sus componentes fundamentales, elementos de recursos de coping (Andersen y Williams, 1988; Junge, 2000; Williams y Andersen, 1998), y diferentes estudios han mostrado el importante papel del afrontamiento en este ámbito (Berengüí et al., 2011; Hanson, McCullagh y Tonymon, 1992; Maddison y Prapavessis, 2005; Ortín, 2008). Stone, Helder y Schneider (1987) y Williams (1996) mantienen que el afrontamiento de las situaciones puede tener un efecto directo sobre las lesiones, al inocular a una persona contra la vulnerabilidad a la lesión, o también un efecto indirecto al moderar o mediar en la respuesta al estrés, los efectos negativos de los estresores y rasgos de personalidad del individuo. Por ello, afirmamos el efecto positivo que puede conllevar un mejor afrontamiento de eventos negativos en el contexto deportivo, con cifras inferiores de lesiones en aquellos individuos que presentan mayores puntuaciones o control de la variable.

En conclusión, el presente estudio muestra un mayor padecimiento de lesiones en deportistas de mayor edad y nivel de rendimiento, y como aquellos con menores niveles en Estabilidad Emocional y Control de Afrontamiento Negativo, y altas en la Ansiedad, registran un mayor número de lesiones. Los deportistas que no registraron lesión alguna se diferencian de los lesionados por mayores niveles de Estabilidad Emocional, Privacidad y Apertura al cambio, Control de Afrontamiento, y menores de Ansiedad, existiendo correlaciones estadísticamente significativas entre el número de lesiones y puntuaciones elevadas en Estabilidad Emocional, Tensión, Aprensión, Ansiedad, y ansiedad rasgo competitiva. Estos hallazgos respaldan la necesidad de seguir profundizando en la investigación de la personalidad y las características psicológicas asociadas a las lesiones, con el objetivo de detectar los deportistas con un mayor riesgo de padecer lesiones. Parece incuestionable la enorme necesidad de determinar los factores antecedentes de las lesiones deportivas, tanto en plano físico como en el psicológico. La identificación de los antecedentes a la lesión y su control podrían conducir, tanto a los deportistas como a entrenadores, dirigentes deportivos, y especialistas de otras áreas de las Ciencias del Deporte, al desarrollo de estrategias que posibiliten la planificación, diseño e implementación de programas preventivos dirigidos a la reducción del riesgo de padecer lesiones deportivas (Devantier, 2011; Keats, Emery y Finch, 2012). 
Es por ello que son necesarios, como sugerencia para futuros trabajos, un mayor número estudios que impliquen extensas muestras de deportistas, de múltiples disciplinas de competición y rangos de edad más amplios. De igual modo, estudios futuros deben seguir ahondando en el papel de las variables de personalidad del deportista, haciendo uso de escalas de medida como las aquí incluidas y otras diferentes (Chan y Hagger, 2012), con el fin de poder alcanzar una mayor comprensión del fenómeno.

\section{Referencias}

Abenza, L., Olmedilla, A., Ortega, E. y Esparza, F. (2009). Lesiones y factores psicológicos en futbolistas juveniles. Archivos de Medicina del Deporte, 132, 280-288.

Andersen, M. B. y Williams, J. M. (1988). A model of stress and athletic injury: Prediction and prevention. Journal of Sport and Exercise Psychology, 104n(3), 294-306.

Andersen, M, B. y Williams, J. M. (1999). Athletic injury, psychosocial factors and perceptual changes during stress. Journal of Sports Science, 17, 735-751.

Berengüí, R. (2011). Personalidad, características psicológicas y lesiones en el deporte de competición: Análisis de relaciones e incidencia en jóvenes deportistas. Tesis Doctoral no publicada. Murcia: Universidad de Murcia.

Berengüí, R. y Garcés de Los Fayos, E. J. (2008). Lesiones y psicología en tecnificación deportiva. I Simposio de Psicología del Deporte de la UCAM. Murcia: Universidad Católica San Antonio.

Berengüí, R. y Garcés de Los Fayos, E. J. (2010). Ansiedad y lesiones deportivas: Estudio correlacional. Congreso Nacional de Psicología de la Actividad Fisica y el Deporte. Madrid: SEPD.

Berengüí, R., Garcés de los Fayos, E. J. y Almarcha, J. (2008). Características psicológicas y su relación con las lesiones en jóvenes deportistas. En J. Díaz, I. Díaz y J. Dosil (Eds.), La Psicología del Deporte en Iberoamérica: Consolidando la Psicología del Deporte Iberoamericana. Torrelavega: SIPD.

Berengüí, R., Garcés de Los Fayos, E. J., Almarcha, J. y Ortega, E. (2010). Lesiones y personalidad en el deporte de competición. Revista Iberoamericana de Fisioterapia y Kinesiología, 13(1), 10-16.

Berengüí, R., López-Gullón, J. M., Garcés de Los Fayos, E. J. y Almarcha, J. (2011). Factores psicológicos y lesiones deportivas en lucha olímpica y taekwondo. E-balonmano.com: Revista de Ciencias del Deporte, 7(Supl.), 9198.

Blackwell, B. y McCullagh, P. (1990). The relationship of athletic injury to life stress, competitive anxiety and coping resources. Atbletic Training, 25(1), 23-27.

Brewer, B. W. (2001). Psychology of sport injury rehabilitation. En R.N. Singer, H.A. Hausenblas y C.M. Janelle (Eds.), Handbook of Sport Psychology. Nueva York: John Wiley \& Sons.

Brewer, B. W. (2009). Injury prevention and rehabilitation. En B.W. Brewer (Ed.), Sport Psychology. Handbook of Sports Medicine and Science. Oxford: John Wiley \& Sons.

Brown, R. B. (1971). Personality characteristics related to injuries in football. The Research Quarterly, 42(2), 133-138.

Cassidy, C. M. (2006). Understanding Sport-Injury Anxiety. Athletic Therapy Today, 11(4), 57-58

Cattell, R. B., Cattell, A. K. S. y Cattell, H. E. P (1995). 16PF5. Adaptación Española. Madrid: TEA Ediciones.

Cattell, H. E. y Schuerger, J. M. (2003). Essentials of 16PF Assessment. Hoboken: John Wiley \& Sons.

Chan, D. y Hagger, M. S. (2012). Transcontextual Development of Motivation in Sport Injury Prevention Among Elite Athletes. Journal of Sport and Exercise Psychology, 34, 661-682.

Clarke, K. S. y Miller, S. J. (1977). The National Athletic Injury/Illness Reporting System (NAIRS). Proceedings of the Second National Sports Safety Congress. Washington DC: American Alliance for Healthy, Physical Education, Recreation and Dance, 41-45.

Devantier, C. (2011). Psychological Predictors of Injury among Professional Soccer Players. Sport Science Review, 20(5-6), 5-36.
Como limitaciones del estudio debemos señalar la naturaleza de su diseño, el cual restringe la comprensión de las relaciones causales entre variables. También el uso de un cuestionario de personalidad, el 16PF-5, que si bien ha sido ampliamente utilizado en multitud de estudios y contextos, no es propio del ámbito deportivo. Respecto a la población estudiada, con unas características especiales como las de deportistas de tecnificación deportiva, los resultados son limitados a la franja de edad de los mismos.

Dvorak, J. y Junge, A. (2000). Football injuries and physical symptoms: A review of the literature. The American Journal of Sports Medicine, 28(5S), S3S9.

García, A. P. y Aragües G. M. (1998). Sport injuries and level of anxiety in soccer players. Medicina Clínica, 111(2), 45-48.

Gimeno, F., Buceta, J. M. y Pérez-Llantada, M. C. (2007). Influencia de las variables psicológicas en el deporte de competición: evaluación mediante el Cuestionario de Características Psicológicas relacionadas con el Rendimiento Deportivo. Psicothema, 19(4), 667-672.

Hanson, S. J., McCullagh, P. y Tonymon, P. (1992). The relationship of personality characteristics, life-stress, and coping resources to athletic injury. Journal of Sport and Exercise Psychology, 14(3), 262-272.

Hernández, A. (2006). Un cuestionario para la evaluación psicológica de la ejecución deportiva: Estudio complementario entre TCT y TRI. Revista de Psicología del Deporte, 15(1), 71-93.

Hootman, J. M., Dick, R. y Agel, J. (2007). Epidemiology of collegiate injuries for 15 sports: Summary and recommendations for injury prevention initiatives. Journal of Atbletic Training, 42(2), 311-319.

Ivarsson, A. y Johnson, U. (2010). Psychological factors as predictors of injuries among senior soccer players. A prospective study. Journal of Sports Science and Medicine, 9, 347-352.

Jackson, D. W., Jarrett, H., Bailey, D., Kausek, J., Swanson, M. J. y Powell, J. W. (1978). Injury prediction in the young athlete: A preliminary report. The American Journal of Sports Medicine, 6(1), 6-12.

Johnson, U. (2011). Athletes' experiences of psychosocial risk factors preceding injury. Qualitative Research in Sport, Exercise and Health, 3(1), 99115.

Johnson, U., Ekengren, J. y Andersen, M. B. (2005). Injury prevention in Sweden. Helping soccer players at risk. Journal of Sport and Exercise Psychology, 27, 32-38.

Johnson, U. y Ivarsson, A. (2011). Psychological predictors of sport injuries among junior soccer players. Scandinavian Journal of Medicine and Science in Sport, 21, 129-136.

Junge, A. (2000). The influence of psychological factors on sports injuries: Review of the literature. The American Journal of Sports Medicine, 28(5), S10-15.

Junge, A. y Dvorak, J. (2004). Soccer injuries. A review on incidence and prevention. Sports Medicine, 34(13), 929-938.

Karson, M., Karson, S. y O’Dell, J. (2003). 16 PF-5. Una guia para su interpretación en la práctica clínica. Madrid: TEA Ediciones.

Kazemi, M. y Pieter, W. (2004). Injuries at a Canadian National Taekwondo Championships: a prospective study. BMC Musculoskeletal Disorders, 5 , 22

Keats, M. R., Emery, C. A. y Finch, C. F. (2012). Are We Having Fun Yet? Fostering Adherence to Injury Preventive Exercise Recommendations in Young Athletes. Sports Medicine, 42(3), 176-181.

Kolt, G. S. (2004). Psicología de la lesión y la rehabilitación. En G.S. Kolt, L. Snyder-Mackler y P. Renström, Fisioterapia del deporte y el ejercicio. Madrid: Elsevier.

Krasnow, D., Mainwaring, L., y Kerr, G. (1999). Injury, stress, and perfectionism in young dancers and gymnasts. Journal of Dance Medicine and Science, 3, 51-58.

Lazarus, R. S. y Folkman, S. (1984). Stress, appraisal and coping. Nueva York: Springer. 
Loehr, J. E. (1986). Mental toughness training for sports: Achieving atbletic excellence. Lexington: Stephen Greene Press.

Maddison, R. y Prapavessis, H. (2005). A psychological approach to the predic-tion and prevention of athletic injury. Journal of Sport and Excercise Psychology, 27, 289-310.

Martens, R. (1977). Sport Competition Anxiety Test. Champaign: Human Kinetics.

Martens, R., Vealley, R. y Burton, D. (1990). Competitive anxiety in sport. Champaign: Human Kinetics.

Meeuwisse, W. H., Tyreman, H., Hagel, B. y Emery, C. (2007). A dynamic model of etiology in sport injury: the recursive nature of risk and causation. Clinical Journal of Sports Medicine, 17(3), 215-219.

Noh, Y. E. (2005). Psychosocial interventions for the prevention of injury in dance. Tesis Doctoral. Melbourne: Victoria University.

Olmedilla A. (2005). Factores psicológicos y vulnerabilidad a las lesiones deportivas: Un estudio correlacional. Murcia: Quaderna.

Olmedilla, A., Andreu, M. D., Ortín, F. J. y Blas, A. (2009). Ansiedad competitiva, percepción de éxito y lesiones: un estudio en futbolistas. Revista Internacional de Medicina y Ciencias de la Actividad Física y el Deporte, 9(33), 51-66.

Olmedilla, A., Ortega, E., Abenza, L. y Boladeras, A. (2011). Lesiones deportivas y Psicología: Una revisión (2000-2009). Cuadernos de Psicología del Deporte, 11(1), 45-57.

Olmedilla, A., Prieto, J. G. y Blas, A. (2011). Relaciones entre estrés psicosocial y lesiones deportivas en tenistas. Universitas Psychologica, 10(3), 909922.

Ortín, F.J. (2008). Factores psicológicos y socio-deportivos y lesiones en jugadores de fútbol semiprofesionales y profesionales. Tesis Doctoral no publicada. Murcia: Universidad de Murcia.

Passer, M. W. y Seese, M. D. (1983). Life-stress and athletic injury: Examination of positive and negative events and three moderator variables. Journal of Human Stress, 9, 11-16.

Petrie, T. A. (1993). Coping skills, competitive trait anxiety, and playing status: Moderating effects on the life stress-injury relationship. Journal of Sport and Exercise Psychology, 15, 261-274.

Pieter. W. y Zemper, E. D. (1999). Competition injuries in adult taekwondo athletes. 5th IOC World Congress on Sports Sciences with the Annual Conference of Science and Medicine in Sport. Sidney.

Rae, K. y Orchard, J. (2007). The Orchard Sports Injury Classification System (OSICS) Version 10. Clinical Journal of Sport Medicine, 17(3), 201-204.

Rogers, T. M. y Landers, D. M. (2005). Mediating effects of peripheral vision in the life event stress/athletic injury relationship. Journal of Sport and Exercise Psychology, 27, 271-288.

Russell, M. T. y Karol, D. L. (2005). 16 PF-5. Manual. Madrid: TEA Ediciones.

Schneider, J. C. (2006). Emotional sequelae of sports-related injuries: Concussive and orthopedic injuries. Tesis Doctoral. Philadelphia: Drexel University.
Short, S. E., Reuter, J., Brandt, J., Short, M. W. y Kontos, A. P. (2004). The relationships among three components of perceived risk of injury, previous injuries and gender in contact athletes. Atbletic Insight, 6(3), 78-85.

Smith, R. E., Ptacek, J. T. y Smoll, F. L. (1992). Sensation seeking, stress, and adolescent injuries: A test of stress-buffering, risk-taking, and coping skills hypotheses. Journal of Personality and Social Psychology, 62, 10161024.

Smith, R. E., Ptacek, J. T. y Patterson, E. (2000). Moderator effects of cognitive and somatic trait anxiety on the relation between life stress and physical injuries. Anxiety, Stress and Coping, 13(3), 269-288.

Smith, R. E., Smoll, F. L. y Schutz, R. W. (1990). Measurement and correlates of sport-specific cognitive and somatic trait anxiety: The Sport Anxiety Scale. Anxiety, Stress and Coping, 2(4), 263-280.

Steffen, K., Pensgaard, A. M. y Bahr, R. (2009). Self-reported psychological characteristics as risk factors for injuries in female youth football. Scandinavian Journal of Medicine and Science in Sports, 19, 442-451.

Stephan, Y., Deroche, T., Brewer, B. W., Caudroit, J. y La Scanff, C. (2009). Predictors of Perceived Susceptibility to Sport-Related Injury among Competitive Runners: The Role of Previous Experience, Neuroticism, and Passion for Running. Applied Psychology, 58(4), 672-687.

Stevenson, M. R., Hamer, P., Finch, C. F., Elliot, B. y Kresnow, M. J. (2000) Sport, age, and sex specific incidence of sports injuries in Western Australia. British Journal of Sports Medicine, 34(3), 188-194.

Stone, A. A., Helder, L. y Schneider, M. S. (1987). Coping with stressful events: Coping dimensions and issues. En L.H. Cohen (Ed.), Life-events and Psychological Functioning. Londres: Sage Publications.

Taimela, S., Osterman, L., Kujala, U. M., Lehto, M., Korhonen, T. y Alaranta, H. (1990). Motor ability and personality with reference to soccer injuries. The Journal of Sports Medicine and Physical Fitness, 30(2), 194-201.

Valliant, P.M. (1981). Personality and injury in competitive runners. Perceptual and Motor Skills, 53(1), 251-253.

Williams, J. M. (1996). Stress, coping resources, and injury risk. International Journal of Stress Management, 3(4), 209-221.

Williams, J. M. y Andersen, M. B. (1998). Psychosocial antecedents of sport injury: review and critique of the stress and injury model. Journal of $A p$ plied Sport Psychology, 10, 5-25.

Williams, J. M. y Andersen, M. B. (2007). Psychosocial antecedents of sport injury and interventions for risk reduction. En G. Tenenbaum y R.C. Eklund, (Eds.), Handbook of Sport Psychology. Nueva York: John Wiley \& Sons.

Yatabe, K., Fujiya, H., Kato, H., Seki, H., Kohno, T., Kumazawa, Y. y Aoki, H. (2005). Personality and emotional responses to sports injury in U-14 soccer players who participated in the JFA elite-program. Japanese Journal of Clinical Sports Medicine, 13(2), 246-255.

Zetaruk, M. N., Violán, M., Zurakowski, D. y Micheli, L. J. (2005). Injuries in martial arts: a comparison of five styles. British Journal of Sports Medicine, 39, 29-33.

(Articulo recibido: 4-6-2012; revisado: 8-1-2013; aceptado: 14-1-2013) 


\section{Anexo. Cuestionario de lesiones}

DATOS PERSONALES Y DEPORTIVOS

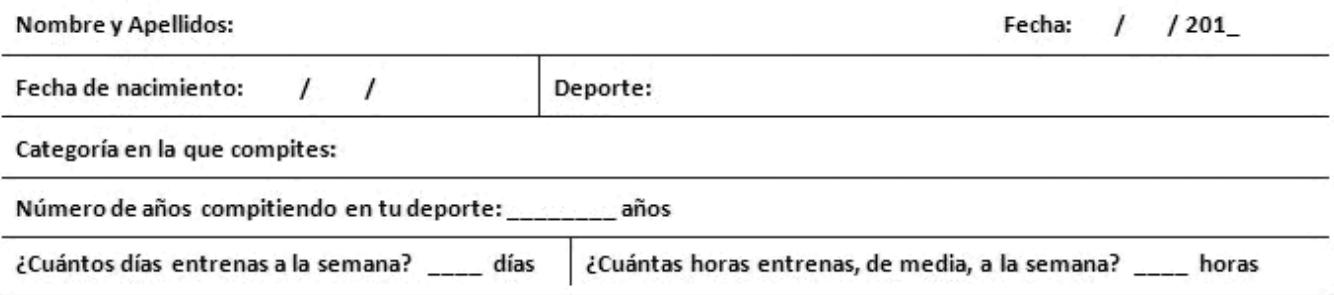

Número de lesiones en las últimas dos temporadas: lesiones

Señala cuales han sido las lesiones que has sufrido, teniendo en cuenta:

- Lesión: nombre o tipo de lesión específica (por ejemplo: esguince de tobillo, sobrecarga lumbar, tendinitis, fractura, etc.). También indica el mes y el año en que tuvo lugar, y si la lesión se originó en entrenamiento o competición.

- Recuperación: tiempo transcurrido hasta tu recuperación total (aproximadamente cuantos días, semanas o meses). Si aun no te has recuperado completamente, anótalo en la casilla correspondiente.

- Causa: cual crees que fue la causa/s de la lesión (por ejemplo: te lesionaste tu solo, técnica inadecuada, el comportamiento de otros, exceso o falta de entrenamiento, mala equipación, golpe con rival, otras causas, etc.).

\begin{tabular}{|c|c|c|c|c|}
\hline Lesión 1 & Fecha & Mes: & Año: & ¿ En entrenamiento $\square$ En competición \\
\hline Recuperación & \multicolumn{2}{|c|}{ __días/__semanas/__meses } & \multicolumn{2}{|c|}{ _ Continuolesionado/a, desde hace: __días/__semanas/_meses } \\
\hline Causa/s & & & & \\
\hline
\end{tabular}

\begin{tabular}{|c|c|c|c|c|}
\hline \multirow{2}{*}{ Lesión 2} & & & & \\
\hline & Fecha & Mes: & Año: & 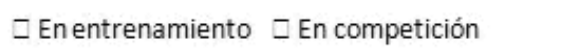 \\
\hline Recuperación & \multicolumn{2}{|c|}{ __días/__semanas/__meses } & \multicolumn{2}{|c|}{ _ Continuo lesionado/a, desdehace:__días/__semanas/_meses } \\
\hline \multicolumn{5}{|l|}{ Causa/s } \\
\hline
\end{tabular}

\begin{tabular}{|c|c|c|c|c|}
\hline Lesión 3 & Fecha & Mes: & Año: & ־ En entrenamiento $\square$ En competición \\
\hline Recuperación & \multicolumn{2}{|c|}{ __dias/__semanas/__meses } & \multicolumn{2}{|c|}{ _ Continuolesionado/a, desdehace:__dias/__semanas/_meses } \\
\hline Causa/s & & & & \\
\hline
\end{tabular}

